\title{
Impact of chemotherapy on the expression of claudins and cadherins in invasive breast cancer
}

\author{
HELENA SKÁLOVÁ ${ }^{1}$, NIKOLA HÁJKOVÁ ${ }^{1}$, BARBORA MAJEROVÁ ${ }^{2}$, \\ MICHAELA BÁRT ${ }^{1}$, CTIBOR POVÝŠIL ${ }^{1}$ and IVANA TICHÁ ${ }^{1}$ \\ ${ }^{1}$ Institute of Pathology, First Faculty of Medicine, Charles University and General University Hospital in Prague, \\ 12800 Prague; ${ }^{2}$ First Faculty of Medicine, Charles University, 12108 Prague, Czech Republic
}

Received January 22, 2019; Accepted July 5, 2019

DOI: $10.3892 / \mathrm{etm} .2019 .7930$

\begin{abstract}
The importance of the expression profile of claudins in the molecular classification of breast cancer (BC) is currently under investigation. Claudins, together with cadherins, serve an important role in the epithelial-mesenchymal transition and influence the chemosensitivity of cancer cells. Adjuvant chemotherapy is administered following surgical resection in selected cases of BC. Previous neoadjuvant chemotherapy may change the molecular profile of a tumour and subsequently also its chemosensitivity. In the current study, the expression of claudin-1, -3 and -4, E- and N-cadherin and the standard BC biomarkers [oestrogen receptor (ER), progesterone receptor (PR), human epidermal growth factor receptor 2 (HER2) and marker of proliferation $\mathrm{Ki}-67$ (Ki-67)] in formalin-fixed, paraffin-embedded sections from 62 patients with invasive $\mathrm{BC}$ was analysed using immunohistochemistry prior to and following neoadjuvant chemotherapy. The results revealed increased expression of claudin-1 $(\mathrm{P}=0.03)$ and decreased expression of claudin-3 $(\mathrm{P}=0.005), \mathrm{PR}(\mathrm{P}<0.001)$ and $\mathrm{Ki}-67$ $(\mathrm{P}=0.01)$ following the neoadjuvant therapy. No significant changes in the expression of ER, claudin-4 or E- and $\mathrm{N}$-cadherin were observed following therapy. Furthermore, an association between the expression of claudin-1 and the standard $\mathrm{BC}$ markers $(\mathrm{P}<0.05)$ was identified. A high expression of
\end{abstract}

Correspondence to: Dr Ivana Tichá or Professor Ctibor Povýšil, Institute of Pathology, First Faculty of Medicine, Charles University and General University Hospital in Prague, Studničkova 2, 12800 Prague 2, Czech Republic

E-mail: ivana.ticha@vfn.cz

E-mail: ctibor.povysil@lf1.cuni.cz

Abbreviations: BC, breast cancer; EMT, epithelial-mesenchymal transition; ER, oestrogen receptor; FFPE, formalin-fixed, paraffin-embedded; FISH, fluorescence in situ hybridization; IHC, immunohistochemistry; NST, (invasive breast carcinoma of) no special type; PR, progesterone receptor; TMA, tissue microarray; TNBC, triple-negative breast cancer

Key words: intercellular junctions, carcinogenesis, immunohistochemistry claudin-1 was more frequently observed in the triple-negative $\mathrm{BC}$ cohort than in the cohort with positive ER, PR and/or HER 2 before $(\mathrm{P}=0.04)$ and after chemotherapy $(\mathrm{P}=0.02)$. The expression of $\mathrm{N}$-cadherin was associated with the expression of ER, PR, HER2 and tumour grade $(\mathrm{P}<0.05)$. A positive association between the expression of claudin-3 and E-cadherin $(\mathrm{P}=0.005)$ was observed. No association was found between the expression of $\mathrm{E}$ - and $\mathrm{N}$-cadherin. In conclusion, significant changes in the expression of claudin- 1 and -3 but not in the expression of claudin-4, E- and N-cadherin were observed in samples taken from patients with BC following chemotherapy. These findings indicate that claudins -1 and -3 serve a role in the response of $\mathrm{BC}$ to chemotherapy.

\section{Introduction}

Breast cancer (BC), the most frequent malignant tumour in females, is a disease with a heterogenous genetic background and morphology. The prediction of the biological behaviour of a tumour and its response to therapy currently relies on histological classification of the BC tumour. BC subtyping based on molecular profiling, including intrinsic subtypes luminal A, luminal B, human epidermal growth factor receptor 2 (HER2)-enriched, basal-like and normal-like, may lead to improved prediction of the tumour's response to therapy (1-3). Novel subgroups with features distinct from the previous ones have been proposed, including the claudin-low and -high subtypes $(4,5)$. However, the additional value of this molecular classification in clinical practice remains unclear (6).

The claudin family currently comprises 27 proteins that form the integral backbone of tight junctions, which are key intercellular connections responsible for maintaining tissue homeostasis (7). The combination of claudins, as well as the level of their expression, are tissue specific and various tumours have been reported to exhibit characteristic patterns (8-11). In non-cancerous breast tissue, the duct epithelium expresses high levels of claudin-1, -3 and -4 (8). In breast tumours, the downregulation or complete loss of claudin-1 and the preservation or upregulation of claudin-3 and -4 were commonly observed in previous studies (8-11). There are, however, some exceptions to these findings, particularly the claudin-low and -high molecular subtypes. The claudin-low subtype is characterized by low expression of claudin-3, -4 and -7 and 
E-cadherin, and a high frequency of triple-negative tumours [TNBC; negative oestrogen (ER) and progesterone receptors (PR) and HER2]. The claudin-high subtype is characterized by the high expression of claudin-1 and -4 and ER negativity. The claudin-low and -high subtypes are associated with aggressive tumours that tend to be chemosensitive, while other therapy options like hormonal or anti-HER2 therapy are frequently limited due to their ER- or triple-negativity $(4,5,12,13)$.

Chemotherapy is one of the basic therapeutic approaches to $\mathrm{BC}$. There are selected patients who profit from neoadjuvant chemotherapy, which is administered prior to surgery in order to reduce the extent of primary tumour and lymph node metastases, and therefore to reduce the extent of required surgery (14). Since many patients undergo adjuvant chemotherapy following the surgical resection of $\mathrm{BC}$, a question arises as to how a possible previous neoadjuvant chemotherapy changes the expression profile of a tumour and whether such changes influence the response to adjuvant chemotherapy.

Claudins have been previously investigated as potential new predictive markers of $\mathrm{BC}(4,5,15)$. Additionally, due to their easily accessible extracellular receptor domains and their involvement in carcinogenesis, claudins are promising targets for novel therapeutic tools, particularly for chemoresistant cancer (16-18). The exact role of claudins in carcinogenesis remains unclear and seems to be very complex, involving proliferation, differentiation and apoptosis on multiple levels $(9,10,19)$. An increasing body of evidence suggests that they serve a role in the epithelial-mesenchymal transition (EMT), during which epithelial neoplastic cells gain a mesenchymal-like phenotype through disruption of intercellular junctions, loss of cell polarity and reorganisation of the cytoskeleton $(12,20,21)$. This process facilitates the growth of a tumour, its invasion into surrounding tissues and metastasis. Furthermore, EMT is thought to be partially responsible for cancer chemoresistance (22). During EMT, intercellular junctions not only come apart but also undergo regulatory changes including a cadherin switch, where epithelial cells loose E-cadherin expression and begin to express $\mathrm{N}$-cadherin, which is typically expressed in tissues of mesodermal origin (23). Even though the expression of E-cadherin is not always entirely lost, a cadherin switch is generally considered to be a marker of EMT $(24,25)$. Claudin-1 facilitates collective migration, by which neoplastic cells detach from the tumour mass at the leading front and penetrate into the surrounding tissues either through partial EMT mechanisms or independently of EMT (26). Claudin-3 and -4 maintain the epithelial phenotype of a cell by modulating the expression of major EMT markers, mainly maintaining the expression of E-cadherin, which is usually highly expressed by the most common type of $\mathrm{BC}$, invasive carcinoma of no special type (NST, ductal) (21). Claudins serve a role in the regulation of cancer stem cells or tumour-initiating cells with self-renewal potential. Therefore, it is possible that they may contribute to drug resistance and tumour recurrence after initial therapy $(12,20,22,27)$.

To the best of our knowledge, this is the first study to investigate the routinely assessed markers of BC such as ER, PR, HER2 and Ki-67, as well as the changes in expression of the intercellular junction proteins claudin-1, -3 and -4, E- and $\mathrm{N}$-cadherin following neoadjuvant chemotherapy. An improved understanding of their expression following chemotherapy may contribute to the elucidation of their role in tumour response to chemotherapy. Furthermore, the current study investigated the associations between the expression of claudins, cadherins, standard BC biomarkers, tumour grade and the extent of histological regressive changes after chemotherapy that may reveal the role of claudins in BC carcinogenesis and EMT.

\section{Materials and methods}

Tissue samples. Formalin-fixed, paraffin-embedded (FFPE) tissue samples of invasive breast carcinoma NST from 62 patients who underwent neoadjuvant chemotherapy between January 2006 and June 2015 were selected from the archive of the Institute of Pathology of the General University Hospital and the First Faculty of Medicine of The Charles University in Prague, Czech Republic. All patients were females whose age at the first biopsy ranged from 23 to 70 years (mean, 53.5 years). The tumour tissue was obtained from a diagnostic core needle biopsy before treatment, and from the definitive resection of the residual tumour following neoadjuvant chemotherapy using mostly anthracyclines or taxanes, in combination with the HER2-antibody trastuzumab in HER2-positive tumours. Patient and tumour characteristics are summarized in Table I. In mastectomy specimens, optimal fixation was ensured by scoring the tissue with parallel incisions (every $10 \mathrm{~mm}$ of tissue) immediately upon the delivery of the specimen to the Institute of Pathology, allowing equal permeation of the tissue by the fixative. This process prevented suboptimal fixation of the specimen and any subsequent alterations in the results of immunohistochemistry (IHC) reactions, specifically the presence of false negativity. Such preparation, together with the inking of the resection margins, enabled the preservation of the anatomical context without hindering macroscopic evaluation of the specimen. All specimens were fixed in $10 \%$ neutral buffered formalin for 6-24 h at room temperature and embedded in paraffin wax. Haematoxylin and eosin-stained $3 \mu \mathrm{m}$ sections were subsequently examined by a pathologist using a light microscope. The histological grade was assessed in the two sample sets according to the Nottingham grading system of Elston and Ellis (1). The effect of therapy in the residual tumour tissue was scored according to the Chevallier classification: i) Class I, no residual carcinoma; ii) class II, carcinoma in situ; iii) class III, invasive carcinoma with stromal fibrosis and class IV, no or minimal regressive changes in the invasive carcinoma (28). Only samples containing residual invasive cancer with either marked (class III) or minor signs of regression after chemotherapy (class IV) were selected. Patients with Chevallier class I and II tumour regression following chemotherapy were not included in the current study due to the absence of invasive cancer.

Samples with a sufficient amount of residual tumour were chosen for tissue microarray (TMA). Representative areas of the tumour were marked and their respective FFPE blocks were used for TMA construction based on the haematoxylin and eosin-stained slides. Three or four tissue cores of $2.0 \mathrm{~mm}$ in diameter were drilled from each donor block and implanted into the recipient block using the tissue microarray instrument TMA Master (3DHISTECH Ltd.). 
Table I. Clinicopathological characteristics of 62 patients with invasive breast carcinoma, including the comparison of tumour stage and grade prior to and following neoadjuvant chemotherapy.

\begin{tabular}{ll}
\hline Variable & $\mathrm{N}(\%)$ \\
\hline
\end{tabular}

Age at the time of core needle biopsy, years $<40$

$40-60$

$>60$

18 (29)

Extent of surgery

Mastectomy

Breast-conserving surgery

30 (48)

Neoadjuvant chemotherapy

Doxorubicin, cyclophosphamide

Doxorubicin, cyclophosphamide, docetaxel

Doxorubicin, cyclophosphamide, paclitaxel

Fluorouracil, epirubicin, cyclophosphamide

Letrozole

Paclitaxel

Other combinations

Neoadjuvant anti-HER2 therapy (trastuzumab)

Yes

No

34 (55)

3 (5)

$3(5)$

4 (6)

2 (3)

11 (18)

$54(87)$

Stage prior to therapy

I

II

III

IV

Unknown

39 (63)

16 (26)

$2(3)$

1 (2)

Stage following therapy

I

II

III

IV

Unknown

Stage changes after neoadjuvant chemotherapy

Decreased

Increased

Unchanged

Unknown

$30(48)$

$12(19)$

2 (3)

$3(5)$

$31(50)$

$11(18)$

$17(27)$

Chevallier class ${ }^{\mathrm{a}}$

III

48 (77)

IV

Tumour grade prior to therapy

G1

$\mathrm{G} 2$

G3

33 (53)

$23(37)$

Tumour grade following therapy

G1

G2

G3

Histological type of breast cancer Invasive carcinoma of NST
Table I. Continued.

Variable

$\mathrm{N}(\%)$

Minor histological component in invasive carcinoma NST ${ }^{\mathrm{b}}$

Mixed NST and lobular

Mixed NST and tubular

NST with neuroendocrine features

$1(2)$

NST with micropapillary component

Total

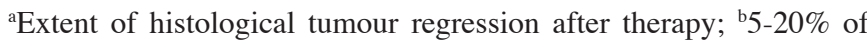
primary tumour mass. NST, no special type.

IHC staining and evaluation. IHC evaluation of expression of all following markers was conducted by a pathologist using a light microscope. The IHC assessment of standard BC biomarkers included HER2, ER, PR and Ki-67. Evaluation of HER2 was performed using HerceptTest (complete kit, original dilution by manufacturer; Agilent Technologies, Inc.; K5204) or rabbit monoclonal anti-human anti-HER2 4B5 PATHWAY antibody (original dilution by manufacturer; Ventana Medical Systems; 790-2991) according to the manufacturers' instructions on the device Ventana BenchMark ULTRA (Ventana Medical Systems). The evaluation of HER2 expression was performed according to the World Health Organization 2012 scoring guidelines (1): i) Negative (score 0 and 1+); ii) weakly positive (2+) and iii) strongly positive (3+) for HER2 protein overexpression. Fluorescence in situ hybridization (FISH) with ZytoLight ${ }^{\circledR}$ HER2/CEN 17 Dual Colour probe (ZytoVision; Z-2077) was performed according to the manufacturer's instructions on samples that were scored by IHC as weakly positive $(2+)$ and also on the samples that were scored as negative (0 and $1+)$ and ER and/or PR negative. For the FISH evaluation, $\geq 20$ cells located in the area of invasive cancer were counted using a fluorescence microscope (magnification, x100 and immerse oil). A positive result was defined as the ratio of HER2: Centromere of chromosome 17 being $\geq 2$. For the evaluation of the other markers, the following antibodies were used according to the manufacturer's instructions: Mouse monoclonal anti-human antibody clone ER-6F11 for the ER receptor (1:50; Novocastra, Leica Microsystems; NCL-L-ER-6F11), two clones PGR-312 and 16 for the PR receptor (1:50; Leica Microsystems Inc.; NCL-PGR-312; ORG-8721) and a mouse monoclonal anti-human clone Mib-1 for the evaluation of Ki-67 (1:50; Agilent Technologies, Inc.; M7240). After that, slides were incubated in N-Histofine Simple Stain Max-Peroxidase (multi) (original dilution by manufacturer, Nichirei Biosciences Inc., 41415) for $30 \mathrm{~min}$ and then in chromogen DAB-3S (original dilution by manufacturer, Nichirei Biosciences Inc., 415194S) for $5 \mathrm{~min}$ at room temperature. The evaluation of these markers was expressed as the percentage of positive cells. The data for ER and PR was categorized into groups as either positive or negative, a positive score was defined as $\geq 1 \%$ of tumour cells showing nuclear staining. A high cell proliferation was defined 62 (100) as $\geq 20 \%$ of Ki-67-positive tumour cells. 
Examination of claudins and cadherins was performed either on whole-tissue sections from the FFPE tissue blocks, which were core needle biopsies and samples containing minimal residual tumour tissue insufficient for the implementation of TMA, or on TMAs. The tissue sections $(4 \mu \mathrm{m})$ were deparaffinised in xylene at room temperature and rehydrated in a graded alcohol series. Antigen retrieval was performed using the heat-induced epitope retrieval technique with a citrate buffer $(\mathrm{pH} 6.0$ for claudin-3 and -4 ; $\mathrm{pH} 9.0$ for claudin-1, E-cadherin and $\mathrm{N}$-cadherin) at $98^{\circ} \mathrm{C}$ for $40 \mathrm{~min}$. Endogenous peroxidase was quenched by $3 \%$ hydrogen peroxide solution in methanol at room temperature for 20 min prior to incubation with the primary antibodies: Polyclonal rabbit anti-human anti-claudin-1 (1:100; Cell Marque Corporation; 359A) and anti-claudin-3 (1:800; Abcam; ab15102), polyclonal goat anti-human anti-claudin-4 (1:100; Santa Cruz Biotechnology, Inc.; sc-17664), monoclonal mouse anti-human anti-E-cadherin (1:100; Thermo Fisher Scientific, Inc.; 18-0223) and anti-N-cadherin (1:300; Agilent Technologies, Inc.; M3613). After that, slides for claudin-1, claudin-3 and E-cadherin were incubated in N-Histofine Simple Stain Max-Peroxidase (multi) (original dilution by manufacturer, Nichirei Biosciences Inc.; 41415) and for claudin-4 in N-Histofine Simple Stain Max-Peroxidase (g) (original dilution by manufacturer, Nichirei Biosciences Inc.; 41416) for $30 \mathrm{~min}$ and then in chromogen DAB-3S (original dilution by manufacturer, Nichirei Biosciences Inc.; 415194S) for $5 \mathrm{~min}$ at room temperature. Slides for $\mathrm{N}$-cadherin were stained by the kit EnVision+ System-HRT (original dilution by manufacturer; Agilent Technologies Inc.; K4006) according to the manufacturers' instructions. Different semi-quantitative scales were used to evaluate the expression of the claudins and cadherins in order to distinguish particular subtypes with a possible impact on tumour biology and/or response to chemotherapy. The evaluation of the expression of claudins was based on a combined score, as previously described $(29,30)$ : Scores 0-3 were used to classify the percentage of positive tumour cells $(0,0 \% ; 1,<25 \% ; 2,25-50 \% ; 3,>50 \%)$ and the intensity of membrane staining $(0,0 ; 1,1+; 2,2+; 3,3+)$. These two scores were subsequently multiplied. In the resulting overall score of $0-9,0$ was considered as negative, 1 or 2 as weakly positive, 3-6 as moderately positive and 9 as strongly positive. Negative and weak positive staining were designated as low expression, while moderate and strong positivity were designated as high expression. For the identification of tumours that could be classified as either molecular claudin-low or -high, the IHC criteria suggested in previous studies were used $(4,31,32)$. The criteria for the claudin-low subgroup included triple negativity and a low or absent expression of at least two of four of the following intercellular junction proteins: Claudin-3, -4, -7 and E-cadherin. Previous studies have added low or absent expression of claudin-1 to these criteria $(31,32)$. The criteria for the claudin-high subgroup included ER negativity and a high expression of claudin-1 and -4 (4). A three-tier scale was applied for the evaluation of E-cadherin $(0,0 \% ; 1,<70 \% ; 2$, $\geq 70 \%$ ). A strong membrane positivity in $>70 \%$ of tumour tissue (score 2) was considered as normal, lower positivity (score 1) or negativity (score 0) were considered as aberrant (33). A four-tier scale was applied for the evaluation of $\mathrm{N}$-cadherin $(0$, $0 \% ; 1,<25 \% ; 2,25-50 \% ; 3,>50 \%$ ). The result was considered as positive when $>1 \%$ of the tumour cells exhibited membrane staining.

Statistical analysis. STATISTICA software (version 10; StatSoft, Inc.) was used for data analysis. The Wilcoxon signed-rank test was used to evaluate the differences in the expression of the markers (ordered categorical) of interest in the paired tumour samples (prior and following treatment). The $\chi^{2}$ test was used to reveal associations between pathological characteristics (dichotomous variables; Tables SI-III). All data used for statistical calculations are in Table SIV. However, the sample set in the current study is limited in size and in the number of cases in the respective categories. Therefore, the outcome of the statistical analysis should be interpreted with caution. All tests were two-sided, and $\mathrm{P}<0.05$ was considered to indicate a statistically significant difference.

\section{Results}

Comparison of the expression of observed markers before and after therapy. The IHC assessment (Table SIV) of the paired tumour samples (before and after chemotherapy) from 62 female patients with invasive breast carcinoma NST revealed a lower expression of PR (median before-/after treatment 25/2; $\mathrm{Z}=3.7 ; \mathrm{P}<0.001$ ) and $\mathrm{Ki}-67$ (median 25/10; $\mathrm{Z}=2.7 ; \mathrm{P}=0.01$ ) following chemotherapy. No significant differences were observed in the expression of E-cadherin, $\mathrm{N}$-cadherin, ER or HER2, or in the frequency of TNBC $(\mathrm{P}>0.05)$ when comparing tumours prior to and following treatment. In 4 cases, the expression of HER 2 changed from negative to positive, and in 3 of these the positive IHC result of $2+$ was confirmed by FISH (the remaining tumour had the positive result of $3+$ determined by IHC; Fig. S1). The tumour grade was not significantly affected following therapy $(\mathrm{P}>0.05)$. The assessment of the histological grade of residual tumours was often limited by the small amount of residual tumour tissue and/or the cytopathic effects of the drugs. The current study included a sample set made up of patients chosen for neoadjuvant chemotherapy, and therefore it included tumours of a higher grade when compared with other studies that analysed cohorts of unsorted patients with BC (34). The residual tumours following chemotherapy displayed either strong or minor regressive changes with fibrosis corresponding to Chevallier class III $(48 / 62 ; 77 \%)$ or class IV (14/62; 23\%), respectively (Fig. 1).

Membrane staining of claudin-1 was punctate and less intense when compared with the more continuous moderate-to-strong staining of claudin-3 and -4 found in the majority of the samples. Cytoplasmic and nuclear staining of claudin-1 appeared in approximately one-third of the samples. In $\sim 20 \%$ of samples, cytoplasmic staining of claudin-3 and -4 was observed. The apicolateral polarity of claudin expression, which is common in non-tumour breast epithelium (8), was absent in the tumour tissue for all three examined claudins (Fig. S2). The expression of claudin-1 increased following neoadjuvant chemotherapy (median before-/after treatment $1.5 / 3 ; \mathrm{Z}=2.2 ; \mathrm{P}=0.03$ ), but a decrease in claudin-3 expression was observed (median 6/5; $\mathrm{Z}=2.8 ; \mathrm{P}=0.005$ ). Furthermore, a reduction in the expression of claudin- 4 following chemotherapy was observed (mean, 7.56 vs. 6.87), but this difference was not statistically significant ( $P>0.05$; Figs. 2 and 3 ). 

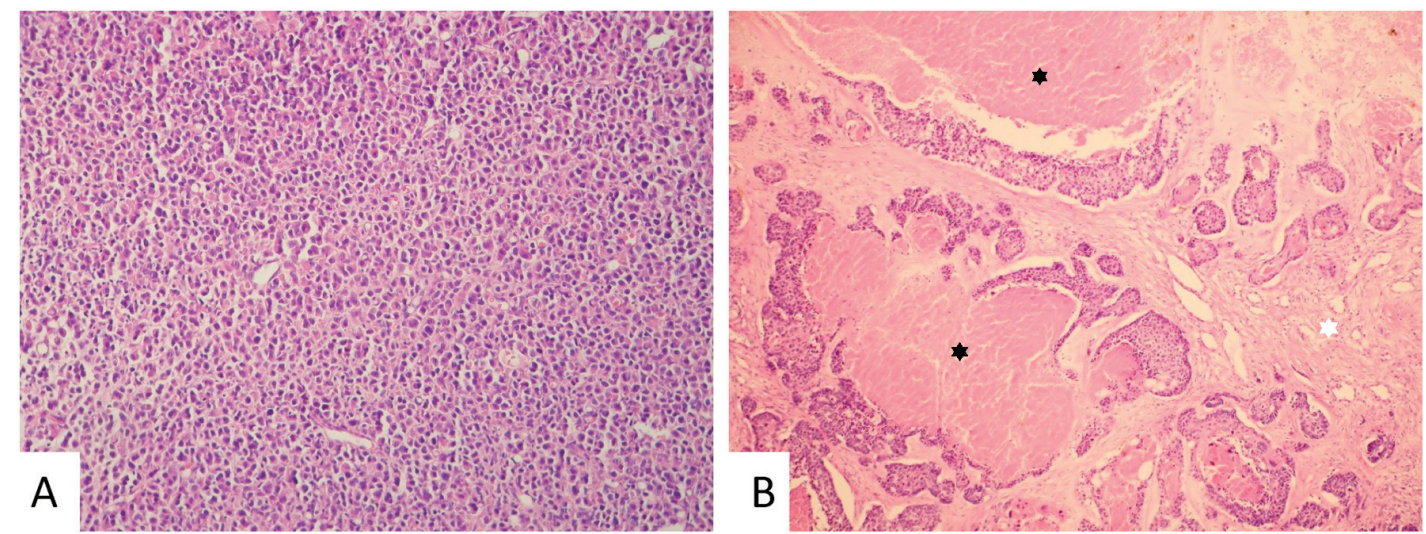

Figure 1. Representative images of invasive breast carcinoma NST prior and following neoadjuvant chemotherapy. (A) Invasive breast carcinoma NST of a solid architecture in a core needle biopsy prior to therapy. Haematoxylin and eosin staining, magnification, x200. (B) Residual carcinoma of a predominantly solid architecture with scattered cribriform pattern with regressive changes [focal necrosis (black asterisks) and fibrosis (white asterisk)] following therapy. Haematoxylin and eosin staining, magnification, x100. NST, no special type.

Table II summarizes simplified dichotomously categorized results from IHC analysis of expression of claudins and other observed markers.

No tumour tissues with low expression of all three claudins (claudin-1, -3 and -4) prior to therapy were observed in the current study; however, four such cases $(4 / 62 ; 6 \%)$ were observed following therapy, all with simultaneous loss of E-cadherin. Only one of these cases was TNBC and matched the suggested criteria for IHC identification of claudin-low subtype. Another case of TNBC in the post-therapy cohort $(1 / 62 ; 2 \%)$ with low expression of claudin-1 and -3 and reduced E-cadherin expression matched the criteria only partially, as the present study did not include the analysis of claudin-7. There were 11 cases $(11 / 62 ; 18 \%)$ pre- and 10 cases $(10 / 62$; $16 \%)$ post-therapy that matched the suggested criteria for the claudin-high subtype. Upregulation of HER2 was observed in $2(2 / 11 ; 17 \%)$ claudin-high tumours pre- and in 1 tumour $(1 / 10$; $6 \%$ ) post-therapy.

Association of the observed markers. A statistical analysis of the association of the expression of claudins and cadherins with other variables was performed (Tables SI-III). However, the results have to be interpreted with caution due to the limited sample set. A negative association between the expression of claudin- 1 and $E R\left[\chi^{2}=5.79\right.$; degrees of freedom $\left.(\mathrm{df})=1 ; \mathrm{P}=0.02\right]$ was observed in tumours prior to chemotherapy but not in the residual tumour tissue following neoadjuvant chemotherapy. Claudin-1 was inversely associated with $\mathrm{PR}\left(\chi^{2}=4.66 ; \mathrm{df}=1\right.$; $\mathrm{P}=0.03)$ and HER2 $\left(\chi^{2}=5.35 ; \mathrm{df}=1 ; \mathrm{P}=0.02\right)$ in the residual tumours following therapy, but not in the core biopsies prior to therapy. TNBC was associated with a high expression of claudin- 1 in tumours prior to $\left(\chi^{2}=4.29 ; \mathrm{df}=1 ; \mathrm{P}=0.04\right)$ and following therapy $\left(\chi^{2}=5.95 ; \mathrm{df}=1 ; \mathrm{P}=0.02\right)$. High expression of claudin-1 was only associated with a high $\mathrm{Ki}-67$ expression following therapy $\left(\chi^{2}=4.98 ; \mathrm{df}=1 ; \mathrm{P}=0.03\right)$. No association between claudin-1 and either $\mathrm{N}$-cadherin or E-cadherin was observed ( $\mathrm{P}>0.05$; Table SI).

Following therapy, the expression of claudin-3 was positively associated with the expression of E-cadherin $\left(\chi^{2}=7.77\right.$; $\mathrm{df}=1 ; \mathrm{P}=0.005)$. No association between claudin-3 and the standard $\mathrm{BC}$ biomarkers or $\mathrm{N}$-cadherin was observed
( $P>0.05)$. A statistical evaluation for claudin-3 before therapy and claudin-4 in both sample sets was not performed due to the unequal distribution of data in the cohorts with high and low expression. Low expression of claudin-3 was only observed in 3 cases and low expression of claudin- 4 was observed in 1 case before therapy and 4 cases after therapy (Table SII).

The expression of $\mathrm{N}$-cadherin had a negative association with $\mathrm{ER}\left(\chi^{2}=6.68 ; \mathrm{df}=1 ; \mathrm{P}=0.01\right)$ and $\mathrm{PR}$ expression $\left(\chi^{2}=4.45\right.$; $\mathrm{df}=1 ; \mathrm{P}=0.04)$ in tumours before therapy and a positive association with HER2 $\left(\chi^{2}=6.40 ; \mathrm{df}=1 ; \mathrm{P}=0.01\right)$ in tumours after therapy. Positive $\mathrm{N}$-cadherin expression was more frequently observed in tumours with a higher grade both before $\left(\chi^{2}=4.45\right.$; $\mathrm{df}=1 ; \mathrm{P}=0.04)$ and after therapy $\left(\chi^{2}=6.46 ; \mathrm{df}=1 ; \mathrm{P}=0.01\right)$. An association between the expression of E-cadherin and standard $\mathrm{BC}$ biomarkers was not observed $(\mathrm{P}>0.05)$. Interestingly, no association between the expression of E-cadherin and $\mathrm{N}$-cadherin was observed $(\mathrm{P}>0.05)$. Although reduction or loss of E-cadherin was observed in 15 tumors before $(15 / 62,24 \%)$ and 18 after therapy $(18 / 62,29 \%)$, and $\mathrm{N}$-cadherin positivity was observed in 15 tumors before $(15 / 62,24 \%)$ and 14 after therapy $(14 / 62,23 \%)$, both of these features simultaneously were detected only in 5 tumours pre- $(5 / 62 ; 8 \%)$ and 4 post$(4 / 62 ; 6 \%)$ therapy (Table SIII).

A larger extent of tumour regression after therapy (Chevallier class III) was associated with higher Ki-67 before treatment $\left(\chi^{2}=5.97 ; \mathrm{df}=1 ; \mathrm{P}=0.02\right)$, when considering only the histological characteristics of the primary tumour and not other clinicopathological data (data not shown). Other markers did not show any association with the histologically assessed tumour regression after therapy $(\mathrm{P}>0.05)$. However, this finding was not conclusive as tumours with a regression of Chevallier class I and II were not included in the current study due to the absence of invasive cancer in these samples.

\section{Discussion}

Claudins are involved in carcinogenesis and cancer progression. Their involvement in the EMT and response to chemotherapy has previously been investigated (12,20-22,26). However, their exact role and relevant regulatory mechanisms remain unclear. The expression pattern of several members 

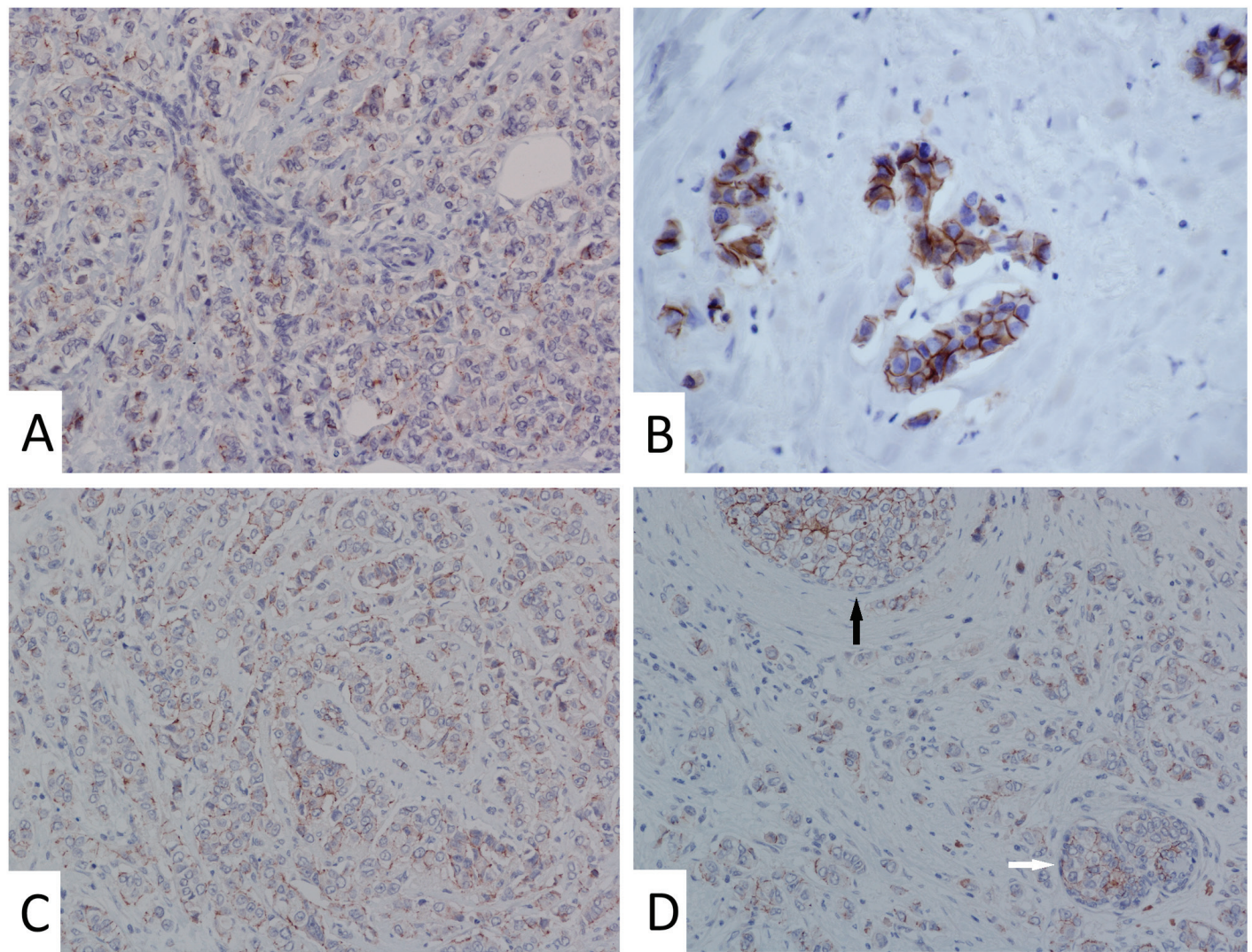

C
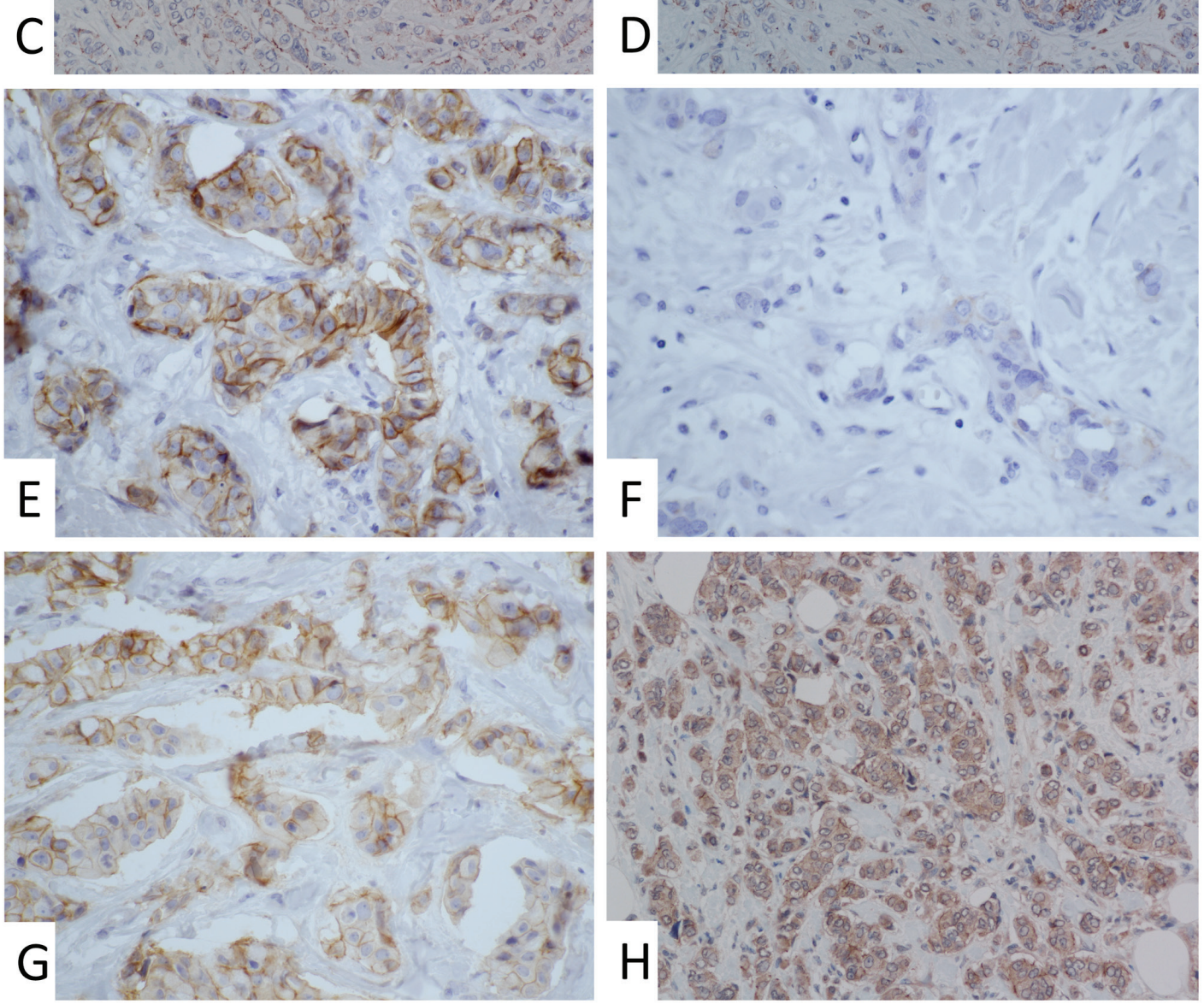

Figure 2. Representative images of changes in the expression of claudins in invasive breast carcinoma no special type following neoadjuvant chemotherapy. (A) Punctate membranous positivity of claudin-1 in a core needle biopsy prior to therapy. Magnification, x200. (B) Strong and continuous membrane staining of claudin-1 in the residual cancer from the same patient. Magnification, x600. (C) Punctate-to-continuous membranous positivity of claudin-1 in a core needle biopsy prior to therapy. Magnification, x200. (D) Similar staining pattern of claudin-1 in the residual cancer from the same patient. Continuous and intense positivity was observed in ductal carcinoma in situ (black arrow) and in the small residual non-tumour duct (white arrow). Magnification, x200. (E) Strong and continuous membranous staining of claudin-3 in a core needle biopsy prior to therapy. Magnification, x600. (F) Faint claudin-3 staining in the residual cancer from the same patient. Magnification, x600. (G) Moderate membranous positivity of claudin-4 in a majority of the tumour cells in a core needle biopsy before therapy (retraction artefacts). Magnification, x600. (H) Similar staining pattern of claudin-4 (comparing membranous positivity only) in the residual cancer from the same patient. Magnification, x200. 
Table II. Immunohistochemical characteristics of invasive breast carcinoma prior and following therapy.

\begin{tabular}{|c|c|c|}
\hline Marker & $\begin{array}{c}\text { Pre therapy, } \\
\mathrm{n}(\%)\end{array}$ & $\begin{array}{c}\text { Post therapy } \\
\mathrm{n}(\%)\end{array}$ \\
\hline \multicolumn{3}{|l|}{ Claudin-1 } \\
\hline High & $25(40)$ & $34(55)$ \\
\hline Low & $37(60)$ & $28(45)$ \\
\hline \multicolumn{3}{|l|}{ Claudin-3 } \\
\hline High & $59(95)$ & $51(82)$ \\
\hline Low & $3(5)$ & $11(18)$ \\
\hline \multicolumn{3}{|l|}{ Claudin-4 } \\
\hline High & $61(98)$ & $58(94)$ \\
\hline Low & $1(2)$ & $4(6)$ \\
\hline \multicolumn{3}{|l|}{ E-cadherin } \\
\hline Normal & $47(76)$ & $44(71)$ \\
\hline Aberrant & $15(24)$ & $18(29)$ \\
\hline \multicolumn{3}{|l|}{$\mathrm{N}$-cadherin } \\
\hline Negative & $47(76)$ & $48(77)$ \\
\hline Positive & $15(24)$ & $14(23)$ \\
\hline \multicolumn{3}{|l|}{ ER } \\
\hline Positive & $45(73)$ & $48(77)$ \\
\hline Negative & $17(27)$ & $14(23)$ \\
\hline \multicolumn{3}{|l|}{ PR } \\
\hline Positive & $39(63)$ & $35(56)$ \\
\hline Negative & $23(37)$ & $27(44)$ \\
\hline \multicolumn{3}{|l|}{ HER2 } \\
\hline Positive & $8(13)$ & 12 (19) \\
\hline Negative & $54(87)$ & $50(81)$ \\
\hline \multicolumn{3}{|l|}{$\mathrm{Ki}-67$} \\
\hline High & $43(70)$ & $25(40)$ \\
\hline Low & $19(30)$ & $37(60)$ \\
\hline Triple-negative breast cancer & $12(19)$ & $10(16)$ \\
\hline
\end{tabular}

The level of expression is categorized high-low, positive-negative or normal-aberrant according to the expected impact on tumour biology and/or response to therapy. ER, oestrogen receptor; PR, progesterone receptor; HER2, human epidermal growth factor receptor 2; Ki-67, marker of proliferation Ki-67.

of the claudin family has been described in numerous types of tumours including breast, ovarian, pancreatic or prostate cancer and can be employed in the diagnostic process namely in gynecological and renal carcinomas or mesothelioma $(29,35,36)$. Analysis of claudin expression has been suggested to enhance the molecular classification of $\mathrm{BC}$ and may therefore affect the indication for chemotherapy in the future, although the use of molecular classification in clinical practice remains questionable $(1,6)$. A large number of patients with BC selected for neoadjuvant therapy receive chemotherapy twice during treatment. The selective pressure of chemotherapy on cancer cells may change the expression profile of a tumour and thereby lead to a loss of sensitivity to anticancer drugs $(37,38)$.

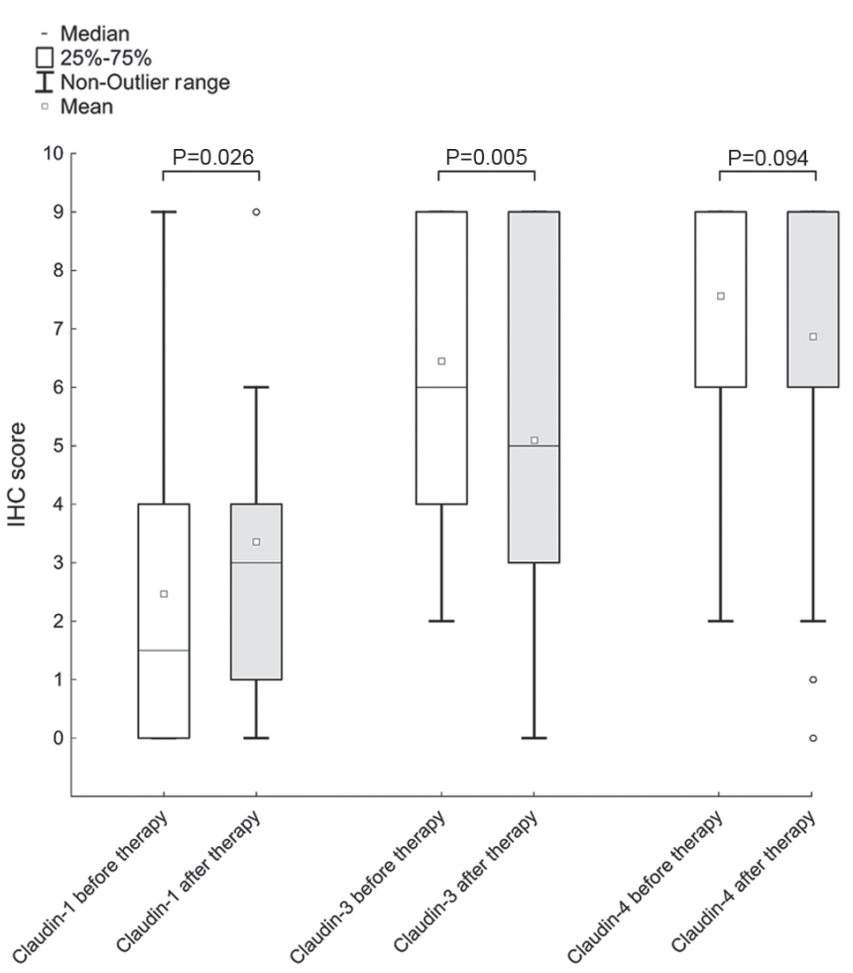

Figure 3. Changes in claudin expression following neoadjuvant chemotherapy. Expression of claudin-1 increased after therapy, while a decrease in expression was observed for claudin-3. Expression of claudin-4 did not change significantly.

Previous studies have compared the expression of the standard IHC BC markers in diagnostic core needle biopsies and surgical specimens in patients who did and did not receive neoadjuvant chemotherapy. In patients who did not receive neoadjuvant chemotherapy, a high concordance was found for all four markers (39-41). However, the majority of studies focusing on patients after chemotherapy revealed substantial changes in these markers (42-51). We observed a decrease of cell proliferation (Ki-67) and PR expression, and only insignificant changes of ER after therapy. This result is in accordance with previous studies that reported similar IHC evaluation of the expression of standard BC markers $(43,49-51)$. The decrease of Ki-67 expression after chemotherapy may be due to the antiproliferative effects of common anticancer drugs $(47,48,52)$. The data obtained in the current study suggested that HER2 expression was unchanged after chemotherapy, which was also reported in a previous study (42). However, this finding is not in agreement with previous studies that described either downregulation (45) or upregulation of HER2 after therapy (53). In other studies, the IHC evaluation of the HER2 status pre- and post- therapy revealed stronger discrepancies, while the status of gene amplification assessed by FISH was reported as rather stable $(44,46)$. In the present study, both techniques (IHC and FISH) were used according to the American Society of Clinical Oncology guidelines (54), with concordant results. However, in certain tumours the areas of HER2 overexpression are only focal and might be missed in the core needle biopsy (55), which may result in the ambiguity of the results reported in literature.

The present study revealed a significant upregulation of claudin-1 and a downregulation of claudin-3 after chemotherapy. Furthermore, claudin-4 expression was downregulated 
but not significantly. The expression of claudin-1 is frequently decreased or lost in cancer cells when compared with the luminal cells of non-tumour breast tissue (30), a feature that was also observed in the current study. Claudin-1 may act as a tumour suppressor or tumour enhancer, depending on cancer type and other not yet well understood conditions $(12,26)$. As the role of claudin-1 is possibly not limited to tight junctions, cytoplasmic or nuclear expression is a common finding $(26,56)$. As a tumour suppressor, its reduced expression may facilitate the EMT and collective migration and may contribute to chemoresistance $(26,57,58)$. However, the current study revealed an increased expression of claudin-1 after therapy, suggesting that other mechanisms may be involved. Our data also showed an association between high expression of claudin-1 and increased rates of cell proliferation.

In the current study, a high expression of claudin-1 was more frequently observed in TNBC irrespective of the administered treatment, which is in concordance with studies that described a higher expression of claudin-1 in basal-like BC, which includes mainly TNBC $(30,59)$. Furthermore, the data obtained in the current study suggested that a high expression of claudin-1 was more common in ER-negative breast tumours before treatment, and in PR-negative or HER2-negative tumours after treatment. A higher expression of claudin-1 in ER-negative compared with ER-positive BC has been previously described $(26,60)$. Additionally, in the current study, positive expression of $\mathrm{N}$-cadherin was more frequently observed in ERor PR-negative BC prior to treatment, and in HER2-positive $\mathrm{BC}$ following treatment. The upregulation of $\mathrm{N}$-cadherin contributes to the invasive phenotype and metastatic potential of moderately-to-poorly differentiated breast carcinomas, which often lose the expression of hormonal receptors and/or overexpress HER2 $(61,62)$. The current study did not reveal an association between claudin-1 and $\mathrm{N}$-cadherin expression, despite the fact that they share some common features in relation to standard BC markers, namely, expression of ER and PR.

The expression of claudin- 3 and -4 is high in the luminal cells of non-tumour breast tissue, and typically remains high in BC (8-11). Concordantly, a high expression of these claudins was found in the majority of breast tumour samples in the current study, despite a decrease in expression after chemotherapy. Claudin-3 and -4 are considered to maintain the epithelial phenotype of epithelial cells by modulating the expression of major EMT proteins, specifically by maintaining the expression of E-cadherin (21). The results obtained in the current study suggest an association between the expression of E-cadherin and claudin-3.

Considering the interactions between claudins and cadherins, the reduced expression of claudin-3 and -4 in neoplastic cells may increase the resistance to chemotherapy. The results obtained in the current study support this theory, as a reduction of the expression of both these markers after therapy was observed. However, previous studies reported conflicting results and may be difficult to interpret in relation to the current study as the majority of those studies focused on ovarian cancer and platinum-based chemotherapy $(57,58,63)$. While platinum-based chemotherapy may be used in the treatment of BC, drugs such as anthracyclines and taxanes are more commonly indicated. Nevertheless, a summary of the aforementioned studies suggests the possibility that a reduced expression of claudin-3 and - 4 increases the resistance to chemotherapy, although the influence of factors such as the type of cancer or chemotherapy used cannot be excluded (12).

Despite the fact that high expression of claudin-3, claudin-4 and E-cadherin is common in invasive breast carcinoma NST, their association with standard BC markers remains unclear. Previous studies detected a slightly higher expression of claudin-3 in ER-positive compared with ER-negative tumours, a higher expression of claudin-4 in ER-negative and basal-like tumours (TNBC) and a more frequent aberrant expression of E-cadherin in ER-negative tumours. However, these associations were not observed in other studies $(30,33,60,64,65)$. The data obtained in the current study were insufficient for statistical evaluation of the association between claudin-4 and any other marker, and only partially sufficient for the evaluation of claudin-3. No associations between claudin-3 and the hormonal receptors, HER2 and Ki-67 were observed. Similarly, E-cadherin was not found to be associated with any of the standard BC markers.

Claudin-low $\mathrm{BC}$ is more frequent among residual tumours after chemotherapy, which is also supported by our experience from the current study $(12,66)$. However, the suggested IHC criteria for the identification of this subgroup do not fully overlap with the molecular claudin-low subtype. Since earlier studies presented claudin-low tumours as mostly TNBC, this feature has been included in the IHC criteria $(5,15)$. Later, however, it was demonstrated that this molecular subtype contains a proportion of ER-positive and non-TNBC tumours, suggesting a large heterogeneity of this subtype (66). The relevance of the claudin-low and -high subgroups remains to be established $(1,6)$.

Assessment of the tumour grade post-chemotherapy has its limitations and must be considered with caution. The results obtained in the current study do not indicate marked changes in tumour differentiation after therapy. The only marker from the studied proteins that reliably associated with tumour grade was $\mathrm{N}$-cadherin, the expression of which is associated with increased invasiveness of poorly differentiated tumours $(61,62)$. The results obtained in the current study did not reveal an association between tumour grade and E-cadherin, the expression of which is more frequently reduced in poorly differentiated carcinomas when compared with well-differentiated invasive breast carcinomas NST $(62,67)$. However, this feature may have been obscured by the limited number of well-differentiated tumours (grade 1) in the current study.

Previous studies reported a wide range $(0-45 \%)$ of aberrant E-cadherin expression in invasive breast carcinoma NST $(33,62,67)$. Positive expression of $\mathrm{N}$-cadherin in invasive breast carcinoma NST reaching $50 \%$ has been reported, which is twice that observed in the current study; however, the studies reporting this only took into account moderately and/or poorly differentiated tumours $(61,62)$. The involvement of both cadherins in the chemotherapy response may be due to their role in the EMT $(68,69)$. The downregulation of E-cadherin and the upregulation of $\mathrm{N}$-cadherin increase chemoresistance $(68,69)$. However, in the current study, the changes in expression of the two cadherins following the therapy were not statistically significant. The upregulation of $\mathrm{N}$-cadherin does not have to be accompanied by the downregulation of E-cadherin, despite 
the already described phenomenon of cadherin switch $(24,25)$. Furthermore, a significant dependence was not observed in the current study. Although E-cadherin downregulation was observed in approximately one-quarter of the examined tumours, and $\mathrm{N}$-cadherin positive expression was observed in a similar proportion of the tumours, only one-third of the tumours exhibited these features simultaneously.

In summary, the current study described significant changes in the expression of claudin-1 and -3 but not in the expression of claudin-4, E- and $\mathrm{N}$-cadherin in $\mathrm{BC}$ following chemotherapy. Moreover, the current study revealed a number of associations between the expressed markers, recently described in other studies $(21,26,30,59,60-62)$, which suggested that such phenomena may frequently occur in $\mathrm{BC}$. The present study revealed that high expression of claudin-1 was observed more frequently in ER- and triple-negative tumours. The association of claudin-1 expression with Ki-67 and HER2 requires further investigation. The association between claudin-3 and E-cadherin corresponds with their role in maintaining epithelial phenotype. The higher frequency of $\mathrm{N}$-cadherin positive expression in poorly differentiated tumours corresponded with the loss of hormonal receptors and HER2 upregulation. The current study was limited by the value of the statistical analyses performed due to the small sample size. Further validations on larger cohorts of patients are required to elucidate the underlying regulatory mechanisms.

\section{Acknowledgements}

The authors would like to thank Mr. Zachary H. K. Kendall (Institute for History of Medicine and Foreign Languages, First Faculty of Medicine, Charles University, Prague, Czech Republic) for the English language corrections.

\section{Funding}

This study was supported by the Ministry of Health(Conceptual Development of Research Organization 64165, General University Hospital in Prague, Prague, Czech Republic), by Charles University (Project Progress Q28/LF1, UNCE 204065 and SVV 260367), by the European Regional Development Fund (project no. BBMRI-CZ; grant no. EF16_013/0001674) and by European Regional Developmental Fund, Operational Program Prague-Competitiveness (Research Laboratory of tumour Diseases; grant no. CZ.2.16/3.1.00/24509).

\section{Availability of data and materials}

The datasets used and/or analyzed during the current study are available from the corresponding author on reasonable request.

\section{Authors' contributions}

HS and CP conceived and designed the study. HS, NH, IT, $\mathrm{MB}$ and $\mathrm{BM}$ collected patient material and clinical data, evaluated the data and performed the analyses. HS, IT and MB drafted the manuscript. MB and CP proofread the manuscript. HS, NH, BM, MB, CP and IT critically reviewed the manuscript and approved the final version of the manuscript.

\section{Ethics approval and consent to participate}

The current study was approved by the Ethics Committee of the General University Hospital in Prague (Prague, Czech Republic) in compliance with the Helsinki Declaration. Additional informed consent signed by patients was not required as the project was approved by Ethics Committee and the data was used for scientific purposes.

\section{Patient consent for publication}

Not applicable.

\section{Competing interests}

The authors declare that they have no competing interests.

\section{References}

1. Lakhani SR, Ellis IO, Schnitt SJ, Tan PH and van de Vijver MJ: WHO classification of tumours of the breast. Fourth edition. IARC, Lyon, 2012.

2. Perou CM, Sørlie T, Eisen MB, van de Rijn M, Jeffrey SS, Rees CA, Pollack JR, Ross DT, Johnsen H, Akslen LA, et al: Molecular portraits of human breast tumours. Nature 406: 747-752, 2000.

3. Sørlie T, Perou CM, Tibshirani R, Aas T, Geisler S, Johnsen H, Hastie T, Eisen MB, van de Rijn M, Jeffrey SS, et al: Gene expression patterns of breast carcinomas distinguish tumour subclasses with clinical implications. Proc Natl Acad Sci USA 98: 10869-10874, 2001.

4. Myal Y, Leygue E and Blanchard AA: Claudin 1 in breast tumorigenesis: Revelation of a possible novel 'claudin high' subset of breast cancers. J Biomed Biotechnol 2010: 956897 , 2010.

5. Prat A,Parker JS, Karginova O, Fan C,Livasy C, Herschkowitz JI, $\mathrm{He} \mathrm{X}$ and Perou CM: Phenotypic and molecular characterization of the claudin-low intrinsic subtype of breast cancer. Breast Cancer Res 12: R68, 2010.

6. Ellis IO, Carder P, Hales S, Lee A, Pinder S, Rakha E and Stephenson T: Pathology reporting of breast disease in surgical excision speciemens incorporating tha dataset for histological reporting of breast cancer. Royal College of Pathologists, 2016.

7. Mineta K, Yamamoto Y, Yamazaki Y, Tanaka H, Tada Y, Saito K, Tamura A, Igarashi M, Endo T, Takeuchi K and Tsukita S: Predicted expansion of the claudin multigene family. FEBS Lett 585: 606-612, 2011.

8. Kulka J and Tökés AM: Claudin expression in breast tumours. Hum Pathol 36: 859-860, 2005.

9. Ding L, Lu Z, Lu Q and Chen YH: The claudin family of proteins in human malignancy: A clinical perspective. Cancer Manag Res 5: 367-375, 2013.

10. Turksen K and Troy TC: Junctions gone bad: Claudins and loss of the barrier in cancer. Biochim Biophys Acta 1816: 73-79, 2011.

11. Singh AB, Sharma A and Dhawan P: Claudin family of proteins and cancer: An overview. J Oncol 2010: 541957, 2010.

12. Kwon MJ: Emerging roles of claudins in human cancer. Int J Mol Sci 14: 18148-18180, 2013.

13. HerschkowitzJI,Simin K, Weigman VJ,Mikaelian I,Usary J,HuZ, Rasmussen KE, Jones LP, Assefnia S, Chandrasekharan S, et al: Identification of conserved gene expression features between murine mammary carcinoma models and human breast tumors. Genome Biol 8: R76, 2007.

14. Buchholz TA, Hunt KK, Whitman GJ, Sahin AA and Hortobagyi GN: Neoadjuvant chemotherapy for breast carcinoma: Multidisciplinary considerations of benefits and risks. Cancer 98: 1150-1160, 2003

15. Prat A and Perou CM: Deconstructing the molecular portraits of breast cancer. Mol Oncol 5: 5-23, 2011.

16. Saeki R, Kondoh M, Kakutani H, Tsunoda S, Mochizuki Y, Hamakubo T, Tsutsumi Y, Horiguchi Y and Yagi K: A novel tumour-targeted therapy using a claudin-4-targeting molecule. Mol Pharmacol 76: 918-926, 2009. 
17. Walther W, Petkov S, Kuvardina ON, Aumann J, Kobelt D, Fichtner I, Lemm M, Piontek J, Blasig IE, Stein U and Schlag PM: Novel Clostridium perfringens enterotoxin suicide gene therapy for selective treatment of claudin-3- and -4-overexpressing tumors. Gene Ther 19: 494-503, 2012.

18. Morin PJ: Claudin proteins in human cancer: Promising new targets for diagnosis and therapy. Cancer Res 65: 9603-9606, 2005.

19. Singh AB and Dhawan P: Claudins and cancer: Fall of the soldiers entrusted to protect the gate and keep the barrier intact. Semin Cell Dev Biol 42: 58-65, 2015.

20. Osanai M, Takasawa A, Murata M and Sawada N: Claudins in cancer: Bench to bedside. Pflugers Arch 469: 55-67, 2017.

21. Lin X, Shang X, Manorek G and Howell SB: Regulation of the epithelial-mesenchymal transition by claudin-3 and claudin- 4 . PLoS One 8: e67496, 2013.

22. Singh A and Settleman J: EMT, cancer stem cells and drug resistance: An emerging axis of evil in the war on cancer. Oncogene 29: 4741-4751, 2010

23. Hazan RB, Phillips GR, Qiao RF, Norton L and Aaronson SA: Exogenous expression of $\mathrm{N}$-cadherin in breast cancer cells induces cell migration, invasion, and metastasis. J Cell Biol 148: 779-790, 2000

24. Nieman MT, Prudoff RS, Johnson KR and Wheelock MJ: $\mathrm{N}$-cadherin promotes motility in human breast cancer cells regardless of their E-cadherin expression. J Cell Biol 147: 631-644, 1999

25. Rai $\mathrm{H}$ and Ahmed J: N-cadherin: A marker of epithelial to mesenchymal transition in tumour progression. Internet J Oncol 10: 1-8, 2014

26. Zhou B, Moodie A, Blanchard AA, Leygue E and Myal Y: Claudin 1 in breast cancer: New insights. J Clin Med 4: 1960-1976, 2015.

27. Baccelli I and Trumpp A: The evolving concept of cancer and metastasis stem cells. J Cell Biol 198: 281-293, 2012

28. Chevallier B, Roche H, Olivier JP, Chollet P and Hurteloup P Inflammatory breast cancer. Pilot study of intensive induction chemotherapy (FEC-HD) results in a high histologic response rate. Am J Clin Oncol 16: 223-228, 1993.

29. Lechpammer M, Resnick MB, Sabo E, Yakirevich E, Greaves WO, Sciandra KT, Tavares R, Noble LC, DeLellis RA and Wang LJ: The diagnostic and prognostic utility of claudin expression in renal cell neoplasms. Mod Pathol 21: 1320-1329, 2008.

30. Lu S, Singh K, Mangray S, Tavares R, Noble L, Resnick MB and Yakirevich E: Claudin expression in high-grade invasive ductal carcinoma of the breast: Correlation with the molecular subtype. Mod Pathol 26: 485-495, 2013.

31. Dias K, Dvorkin-Gheva A, Hallett RM, Wu Y, Hassell J, Pond GR, Levine M, Whelan T and Bane AL: Claudin-low breast cancer; Clinical \& pathological characteristics. PLoS One 12: e0168669, 2017.

32. Gerhard R, Ricardo S, Albergaria A, Gomes M, Silva AR, Logullo ÂF, Cameselle-Teijeiro JF, Paredes J and Schmitt F: Immunohistochemical features of claudin-low intrinsic subtype in metaplastic breast carcinomas. Breast 21: 354-360, 2012 .

33. Kowalski PJ, Rubin MA and Kleer CG: E-cadherin expression in primary carcinomas of the breast and its distant metastases. Breast Cancer Res 5: R217-R222, 2003.

34. Dewis R and Gribbin J: Breast cancer: Diagnosis and treatment: An assessment of need. National Collaborating Centre for Cancer (UK), Cardiff, UK, 2009.

35. Ordóñez NG: Value of claudin-4 immunostaining in the diagnosis of mesothelioma. Am J Clin Pathol 139: 611-619, 2013.

36. Szabó I, Kiss A, Schaff Z and Sobel G: Claudins as diagnostic and prognostic markers in gynecological cancer. Histol Histopathol 24: 1607-1615, 2009.

37. Worsley CM, Mayne ES and Veale RB: Clone war: The evolution of therapeutic resistance in cancer. Evol Med Public Health 2016: 180-181, 2016.

38. Sun D, Dalin S, Hemann MT, Lauffenburger DA and Zhao B: Differential selective pressure alters rate of drug resistance acquisition in heterogeneous tumor populations. Sci Rep 6 36198, 2016.

39. Asogan AB, Hong GS and Arni Prabhakaran SK: Concordance between core needle biopsy and surgical specimen for oestrogen receptor, progesterone receptor and human epidermal growth factor receptor 2 status in breast cancer. Singapore Med J 58: 145-149, 2017.
40. Dekker TJ, Smit VT, Hooijer GK, Van de Vijver MJ, Mesker WE, Tollenaar RA, Nortier JW and Kroep JR: Reliability of core needle biopsy for determining ER and HER2 status in breast cancer. Ann Oncol 24: 931-937, 2013.

41. You K, Park S, Ryu JM, Kim I, Lee SK, Yu J, Kim SW, Nam SJ and Lee JE: Comparison of core needle biopsy and surgical specimens in determining intrinsic biological subtypes of breast cancer with immunohistochemistry. J Breast Cancer 20: 297-303, 2017.

42. Kinsella MD, Nassar A, Siddiqui MT and Cohen C: Estrogen receptor (ER), progesterone receptor (PR), and HER2 expression pre- and post-neoadjuvant chemotherapy in primary breast carcinoma: A single institutional experience. Int J Clin Exp Pathol 5: 530-536, 2012

43. Yin HF, Wang YH, Qin XQ, Zhang H, Li T, Ye JM and Liu YH: Effect of neoadjuvant chemotherapy on histologic grade and expression of biological markers in breast cancer. Zhonghua Zhong Liu Za Zhi 31: 858-862, 2009 (In Chinese).

44. van de Ven S, Smit VT, Dekker TJ, Nortier JW and Kroep JR: Discordances in ER, PR and HER2 receptors after neoadjuvant chemotherapy in breast cancer. Cancer Treat Rev 37: 422-430, 2011.

45. Yoshida A, Hayashi N, Suzuki K, Takimoto M, Nakamura S and Yamauchi H: Change in HER2 status after neoadjuvant chemotherapy and the prognostic impact in patients with primary breast cancer. J Surg Oncol 116: 1021-1028, 2017.

46. Li P, Liu T, Wang Y, Shao S, Zhang W, Lv Y, Yi J and Wang Z: Influence of neoadjuvant chemotherapy on HER2/neu status in invasive breast cancer. Clin Breast Cancer 13: 53-60, 2013.

47. Cabrera-Galeana $P$, Muñoz-Montaño W, Lara-Medina F, Alvarado-Miranda A, Pérez-Sánchez V, Villarreal-Garza C, Quintero RM, Porras-Reyes F, Bargallo-Rocha E, Del Carmen I, et al: Ki67 Changes identify worse outcomes in residual breast cancer tumors after neoadjuvant chemotherapy. Oncologist 23: 670-678, 2018

48. Moazed V, Jafari E, Kalantari Khandani B, Nemati A, Roozdar A and Ben Razavi SA: Prognostic significance of reduction in Ki67 index after neoadjuvant chemotherapy in patients with breast cancer in kerman between 2009 And 2014. Iran J Pathol 13: 71-77, 2018.

49. Dede DS, Gumuskaya B, Guler G, Onat D, Altundag K and Ozisik Y: Evaluation of changes in biologic markers ER, PR, HER 2 and $\mathrm{Ki}-67$ index in breast cancer with administration of neoadjuvant dose dense doxorubicin, cyclophosphamide followed by paclitaxel chemotherapy. J BUON 18: 366-371, 2013.

50. Lee HC, Ko H, Seol H, Noh DY, Han W, Kim TY, Im SA and Park IA: Expression of immunohistochemical markers before and after neoadjuvant chemotherapy in breast carcinoma, and their use as predictors of response. J Breast Cancer 16 : 395-403, 2013.

51. Zhou X, Zhang J, Yun H, Shi R, Wang Y, Wang W, Lagercrantz SB and $\mathrm{Mu} \mathrm{K}$ : Alterations of biomarker profiles after neoadjuvant chemotherapy in breast cancer: Tumor heterogeneity should be taken into consideration. Oncotarget 6: 36894-36902, 2015.

52. Yoshioka T, Hosoda M, Yamamoto $M$, Taguchi $K$, Hatanaka KC, Takakuwa E, Hatanaka Y, Matsuno Y and Yamashita H: Prognostic significance of pathologic complete response and Ki67 expression after neoadjuvant chemotherapy in breast cancer. Breast Cancer 22: 185-191, 2015.

53. Adams AL, Eltoum I, Krontiras H, Wang W and Chhieng DC: The effect of neoadjuvant chemotherapy on histologic grade, hormone receptor status, and HER $2 /$ neu status in breast carcinoma. Breast J 14: 141-146, 2008.

54. Wolff AC, Hammond MEH, Allison KH, Harvey BE, McShane LM and Dowsett M: HER2 testing in breast cancer: American society of clinical oncology/college of American pathologists clinical practice guideline focused update summary. J Oncol Pract 14: 437-441, 2018.

55. Davila E and Amazon K: The clinical importance of the heterogeneity of HER2 neu. Case Rep Oncol 3: 268-271, 2010.

56. Dhawan P, Singh AB, Deane NG, No Y, Shiou SR, Schmidt C, Neff J, Washington MK and Beauchamp RD: Claudin-1 regulates cellular transformation and metastatic behavior in colon cancer. J Clin Invest 115: 1765-1776, 2005.

57. Fortier AM, Asselin E and Cadrin M: Keratin 8 and 18 loss in epithelial cancer cells increases collective cell migration and cisplatin sensitivity through claudin1 up-regulation. J Biol Chem 288: 11555-11571, 2013. 
58. Li M, Balch C, Montgomery JS, Jeong M, Chung JH, Yan P, Huang TH, Kim S and Nephew KP: Integrated analysis of DNA methylation and gene expression reveals specific signaling pathways associated with platinum resistance in ovarian cancer. BMC Med Genomics 2: 34, 2009.

59. Blanchard AA, Ma X, Dueck KJ, Penner C, Cooper SC, Mulhall D, Murphy LC, Leygue E and Myal Y: Claudin 1 expression in basal-like breast cancer is related to patient age. BMC Cancer 13: 268, 2013

60. Blanchard AA, Skliris GP, Watson PH, Murphy LC, Penner C, Tomes L, Young TL, Leygue E and Myal Y: Claudins 1, 3, and 4 protein expression in ER negative breast cancer correlates with markers of the basal phenotype. Virchows Arch 454: 647-656, 2009.

61. Qian X, Anzovino A, Kim S, Suyama K, Yao J, Hulit J, Agiostratidou G, Chandiramani N, McDaid HM, Nagi C, et al: $\mathrm{N}$-cadherin/FGFR promotes metastasis through epithelial-to-mesenchymal transition and stem/progenitor cell-like properties. Oncogene 33: 3411-3421, 2014.

62. ElMoneim HM and Zaghloul NM: Expression of E-cadherin, $\mathrm{N}$-cadherin and snail and their correlation with clinicopathological variants: An immunohistochemical study of 132 invasive ductal breast carcinomas in Egypt. Clinics (Sao Paulo) 66: 1765-1771, 2011.

63. Shang X, Lin X, Manorek G and Howell SB: Claudin-3 and claudin- 4 regulate sensitivity to cisplatin by controlling expression of the copper and cisplatin influx transporter CTR1. Mol Pharmacol 83: 85-94, 2013.
64. Kulka J, Szász AM, Németh Z, Madaras L, Schaff Z, Molnár IA and Tokés AM: Expression of tight junction protein claudin-4 in basal-like breast carcinomas. Pathol Oncol Res 15: 59-64, 2009.

65. Soini Y: Claudins 2, 3, 4, and 5 in Paget's disease and breast carcinoma. Hum Pathol 35: 1531-1536, 2004.

66. Sabatier R, Finetti P, Guille A, Adelaide J, Chaffanet M, Viens P, Birnbaum D and Bertucci F: Claudin-low breast cancers: Clinical, pathological, molecular and prognostic characterization. Mol Cancer 13: 228, 2014.

67. Singhai R, Patil VW, Jaiswal SR, Patil SD, Tayade MB and Patil AV: E-Cadherin as a diagnostic biomarker in breast cancer. N Am J Med Sci 3: 227-233, 2011.

68. Wang W, Wang L, Mizokami A, Shi J, Zou C, Dai J, Keller ET, $\mathrm{Lu} \mathrm{Y}$ and Zhang J: Down-regulation of E-cadherin enhances prostate cancer chemoresistance via Notch signaling. Chin J Cancer 36: 35, 2017.

69. Nakamura T, Kato Y, Fuji H, Horiuchi T, Chiba Y and Tanaka K: E-cadherin-dependent intercellular adhesion enhances chemoresistance. Int J Mol Med 12: 693-700, 2003.

This work is licensed under a Creative Commons Attribution-NonCommercial-NoDerivatives 4.0 International (CC BY-NC-ND 4.0) License. 